\title{
LUMBAR FLEXION MOMENT MESUREMENT USING SEPARATED SEAT: THE INFLUENCE OF SEAT CONDITION
}

\author{
Dong Hyun Kim, ${ }^{1}$ Seo Hyun Kim, ${ }^{1}$ Young Jin Jung, ${ }^{1}$ Han A Lee, ${ }^{1}$ Dong Hyun Hwang, ${ }^{1}$ Bokku Kang, ${ }^{2}$ Young Keun \\ Cho, ${ }^{2}$ Hyung Joo Kim and ${ }^{1}$ Han Sung Kim(hanskim@yonsei.ac.kr) \\ ${ }^{1}$ Department of Biomedical Engineering, Yonsei University, Won-ju, 445-743 \\ ${ }^{2}$ Human Factors \& Devices Research Team, Hyundai Central Advanced Research \& Engineering Institute, Uiwang, \\ 231-111
}

ABSTRACT

The aim of this study was to investigate the effects of driving posture changed by seat condition including seat heights (high, low), seat angles (94, 109 degree), and either with or without lumbar support in separated seat on lumbar flexion moments. Recent trends in auto industry has led the higher vehicle seat comfort, which is one of the most important factors in selecting a car. However, the biomechanical aspect of driving posture while seating on a seat have received less attention. Even though researchers have suggested an ideal posture for drivers to reduce load on L5/S1, there has been no equipment found for measuring lumbar flexion moment in real time as driving posture changes. All 25 participants (between the ages of 27 and 46, $73.5 \pm 11.5 \mathrm{~kg}, 1.74 \pm 0.11 \mathrm{~m}$ ) should have never suffered from musculoskeletal disorders and any kind of orthopedic surgery around the lumbar region. The separated seat is consisted of two parts including seat pan and seat back and the seat pan was connected to the force plates. All participants took a seat on the seat, which was adjusted for seat back angle (94/109 degree according to ground) and height (Top/ Mid.) with lumbar support (presence/absence) in order to induce various driving postures. Reaction forces were collected with force plates (Advanced Mechanical Technologies, Inc., Newton, MA, USA) and the data of marker position were collected by using an eight-infrared-camera and three-dimensional motion analysis system. (Vicon, Oxford Metrics LTD, Oxford, England) The data of L5 location was changed by car seat condition. Seat back reaction force results showed a significant difference in seat back angle with absence lumbar support. ( $\mathrm{P}=0.0215, \mathrm{P}=0.0208)$ Lumbar flexion moments results showed that there was significant difference in seat back. ( $\mathrm{P}=0.0433, \mathrm{P}=0.0005, \mathrm{P}=0.0159, \mathrm{P}=0.0227$ ) There was no significant difference in seat height and lumbar support. These results indicate that the seat back angle may be the main cause of decreased driver's lumbar flexion moment. However, other car seat adjustment factors for lumbar flexion moment does not have any significance $(\mathrm{P}>0.05)$ This study suggested that lumbar flexion moment may be a new method of measuring seat comfort. Further study should be conducted to find out the relationship between spine curve and lumbar flexion moments.

Keywords: Lumbar flexion moment, Separated seat, Driving posture, Biomechanical load, Seat comfort

\section{Introduction}

Recent trends in auto industry has led the higher vehicle seat comfort, which is one of the most important factors in selecting a car. However, the seating ergonomics has received less attention in the biomechanics of automobile than it does in office and factory.

Previous studies have suggested that driving posture is a key factor determining drivers' comfort while driving since most drivers felt discomfort due to their inappropriate driving posture. (Hirao et al, 2006) The inappropriate driving posture could cause biomechanical loads to spinal column. (Vivek D. Bhise, 2012) Biomechanical loads on sitting posture which are classified into musculoskeletal loads and contact loads, caused lumbar disk degeneration, herniation and muscle spasm. (Pope MH et al, 2002)

Researchers suggested that an ideal driver's posture to reduce load on L5/S1 was considered to be a balanced posture that minimizes passive loads. (Donald D. Harrison et al, 2000, R. Zenk et al., 2012) However, any equipment has been found to measure the lumbar flexion moment in real time as the car seat.

The aim of this study was to measure the changes in lumbar flexion moment caused by changing driving posture with seat adjustments. 


\section{Method}

All 25 participants have never suffered from musculoskeletal disorders and any kind of orthopedic surgery around the lumbar region. The participants were between the ages of 27 and 46 . The weight of them be in the range of $73.5 \pm 11.5 \mathrm{~kg}$ and the height be in the range of 1.74 $\pm 0.11 \mathrm{~m}$.

The separated seat which is divided into two parts is consisted of seat pan and seat back. Seat pan was connected with force plates. A reflective marker was placed on the contact point to fifth lumbar vertebra with seat back surface after each trial.

Lumbar flexion moments were calculated by vector cross product. (1)

$$
M_{\text {Lumbar }}=\Delta r_{\text {Pan }} \cdot F_{\text {Pan }}
$$

After than all participants took a seat which was adjusted for seat back angle (94/109 degree according to ground), height (Top/ Mid.) and with or without lumbar support for inducing various driving posture. All participants were requested to maintain their hands to grip at 2 and 10 o'clock of steering wheel and stare at the painted point on the wall in order to simulate the actual driving situation.

The lumbar flexion moments for each seat condition were performed unpaired t-test to acquire statistical significance $(\mathrm{P}$ $<0.05)$.

\section{Results}

Test results showed that there was significant difference in seat back. There was no significant difference in seat height and lumbar support. (Figure 1)

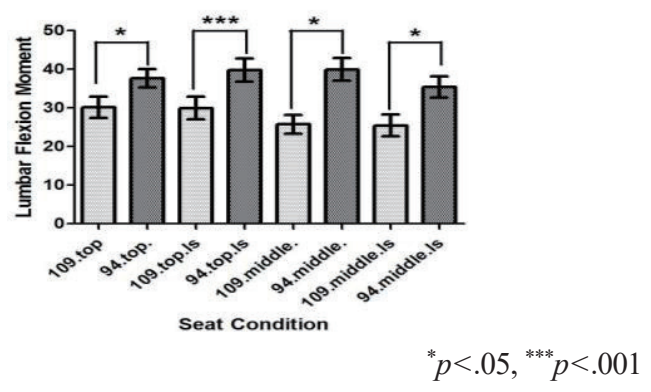

Figure 1 Lumbar flexion moment generated by upper body according to the seat condition.

These results indicate that the seat back angle may be the main cause of decreased driver's lumbar flexion moment. However, other car seat adjustment factors for lumbar flexion moment does not have any significance $(\mathrm{P}>0.05)$.

\section{Conclusion}

Seating condition of seat back angle was the main factor, reducing lumbar flexion moments, while other factors including seat height and lumbar support has had no effects on lumbar flexion moments. This study suggested that lumbar flexion moment may be a new method of measuring seat comfort.

\section{Acknowledgements}

This work was supported by the research program (R7520-16-0005) from the Ministry of Science, ICT and Future Planning of the republic of Korea and the Institute of Information \& communications Technology Promotion of Korea and Hyudai Motor Group.

\section{References}

Akinari Hirao, Satoshi Kitazaki and Nobutoshi Yamazaki, "Development of a new Driving Posture Focused on Biomechanical Loads", $S A E$, 2006-01-1302, 2006.

Vivek D. Bhise, Ergonomics in the Automotive Design Process, CRC Press, 2012.

Pope MH, Goh KL, Magnusson ML, "Spine Ergonomics", Annu Rev Biomed Eng, 4, pp.49-68, 2002

Donald D. Harrison et all, "Sitting Biomechanics, Part II: Optimal Car Driver's Seat and Optimal Driver's Spinal Model”, JMPT, 23(1), pp. $37-47,2000$

R. Zenk, M. Franz, H. Bubb and P. Vink, "Technical note: Spine load in automotive seating”, Applied Ergonomics, 43(2012), pp.290-295, 2012

Andrew P. Claus, Julie A. Hides, G. Lorimer Moseley and Paul W. Hodges, "Is 'ideal' sitting posture real?: Measurement of spinal curves in four sitting postures", Manual Therapy, 14, pp.404-408, 2009 